\title{
Crecimiento de oocitos biderianos en machos adultos de Rhinella marina (Linnaeus, 1758) mediante el uso de hormonas y disruptores endócrinos
}

\author{
Diana Vargas Hurtado y Óscar D. Pérez ${ }^{1}$ \\ ${ }^{1}$ Laboratorio de Biología del Desarrollo y Biología Molecular, Escuela de Ciencias Biológicas, PUCE, \\ Quito, Ecuador. odeperez@puce.edu.ec
}

Recibido: 13, 03, 2012; aceptado: 11, 10, 2012

\begin{abstract}
RESUMEN.- En la región anterior de los testículos de los machos de ciertos taxa de la familia Bufonidae se localiza el órgano de Bidder (OB), un ovario rudimentario no funcional que crece y se desarrolla parcialmente si los testículos son removidos. En esta investigación se analizó el efecto de hormonas y disruptores endócrinos sobre el crecimiento de los oocitos del OB de machos adultos orquidectomizados de la especie Rhinella marina. Machos adultos fueron orquidectomizados y sometidos a dosis diarias de una solución salina, atrazina y progesterona durante un periodo de dos meses y el efecto de dichas substancias fue analizado mediante observaciones en la morfología interna y externa y mediciones del diámetro máximo de los oocitos biderianos de los machos oquidectomizados.

Todos los tratamientos exhibieron un incremento en el diámetro máximo de los oocitos biderianos. Los tratamientos que presentaron oocitos de mayor tamaño y con características correspondientes a estadios de la oogénesis posteriores, fueron el tratamiento con progesterona seguido por el tratamiento con atrazina + progesterona; sin embargo, se obtuvo una baja cantidad de oocitos crecidos. Los resultados sugieren que la hormona progesterona fue la que promovió un mayor crecimiento de los oocitos biderianos de machos orquidectomizados de la especie Rhinella marina.
\end{abstract}

PALABRAS CLAVE: Atrazina, órgano de Bidder, orquidectomización, progesterona, vitelogénesis.

ABSTRACT.- The Bidder's organ is located anterior to the testis of some taxa of bufonid toads ; it is a rudimentary non-functional ovary, which grows and partially develops if the testes are removed. In this research, we analyzed the growing effect of hormones and endocrine disruptors on Bidder's organ (BO) oocytes of orchidectomized adult males of Rhinella marina. Adult males were orchidectomized and subjected to daily doses of saline, atrazine and progesterone solutions for a two months period. The effects of these compounds were tested by observations in the internal and external morphology and measurements of the maximum diameter of the Bidderian follicles.

All treatments showed an increase in the maximum diameter of bidderian oocytes after two months of treatment. Progesterone produced more advanced oogenesis stages than the ones with atrazine + progesterone. However, the number of grown oocytes in both cases was quite low. These results suggest that progesterone is the most suitable for inducing growth in the Bidderian oocytes of orchidectomized Rhinella marina male toads.

KEYWORDS: Atrazine, Bidder's organ, orquidectomy, progesterone, vitellogenesis. 


\section{INTRODUCCIÓN}

El órgano de Bidder (OB) es un ovario vestigial presente en la mayoría de especies de la familia Bufonidae siendo una sinapomorfía de los clados más derivados (Pramuk et al., 2008; Frost et al., 2006; Pramuk, 2006; Duellman y Tueb, 1986). Se desarrolla a partir del primordio gonadal en la etapa larval de los embriones de esta familia. Inicialmente, el primordio gonadal posee características de ovario, las cuales se mantendrán en las hembras hasta el final de su desarrollo. Sin embargo, en los machos, la región posterior del primordio se diferencia en testículo al final de la metamorfosis, mientras que la región anterior mantiene las características de ovario formando el órgano de Bidder (Norris y Lopez, 2011; Falconi et al., 2007; Falconi et al., 2004; Brown et al., 2002).

En los machos de las especies de la familia Bufonidae que poseen OB; como es el caso de las especies del género Bufo, Pedostibes, Nectophrynoides, entre otros (Davis, 1936), la extirpación quirúrgica de uno o de ambos testículos u orquidectomía causará un incremento en el tamaño de dicho órgano (Farias et al., 2002; Pancak-Roessler y Norris, 1991). De igual manera, los oocitos dentro de este órgano crecerán e iniciarán el proceso vitelogénico alcanzando estadios de la oogénesis posteriores (Brown et al., 2002; Cannatella et al., 2001; Pancak-Roessler y Norris, 1991). La ausencia de hormonas masculinas producidas por los testículos, promueve el incremento de tamaño del OB como la iniciación del proceso vitelogénico de sus oocitos; estas hormonas, cuando están presentes, poseen un efecto inhibitorio en el crecimiento de dicho órgano (Brown et al., 2002; Pancak-Roessler y Norris, 1991; Ghosh et al., 1990). El tiempo de crecimiento de los oocitos después de la orquidectomía es bastante lento, alcanzando los estadios II, III y IV de la oogénesis hasta tres meses después de la orquidectomización (Wolpert et al., 2007; Brown et al., 2002; Pancak-Roessler y Norris, 1991).
Durante la oogénesis en anfibios (proceso de formación, crecimiento y maduración de los gametos femeninos) las células germinales femeninas (oogonias) sufren meiosis, la cual está acompañada por pausas o arrestos que se producen en determinados momentos de este proceso (Norris y Lopez, 2011; Gilbert, 2010; Wolpert et al., 2007; Bill, 2002). Un evento importante de la oogénesis es la vitelogénesis (periodo de crecimiento nuclear y citoplasmático que se da después del primer arresto meiótico en diplotene de profase I) en el cual la proteína vitelogenina es sintetizada en el hígado y transportada por el sistema sanguíneo al ovario. Dentro del oocito, esta proteína es convertida en lipoviteina y fosvitina, proteínas que forman parte de las plaquetas de yema, compuesto necesario para la posterior nutrición del embrión (Hausen y Riebesell, 1991; Wallace y Selman, 1990; Wallace y Bergink, 1974).

El sistema endócrino cumple un papel muy importante en la modulación y regulación de la oogénesis. Las hormonas, especialmente las hormonas gonadales, están involucradas en el crecimiento de los oocitos (a través de la estimulación de la vitelogénesis), en la reiniciación del proceso meiótico y en los cambios estructurales del citoplasma y del córtex celular después de la ruptura de la vesícula germinal (Norris y Lopez, 2011; Schuetz, 1974). La hormona folículo estimulante (FSH), por ejemplo, es trascendental dentro del proceso vitelogénico ya que estimula la producción de $17 \beta$-estradiol, el cual a su vez estimula al hígado para que produzca vitelogenina. De igual manera, la hormona luteinizante (LH) estimula producción de progesterona, hormona involucrada en el proceso de maduración nuclear en oocitos completamente desarrollados, oocitos de estadio VI (Norris y Lopez, 2011; Gilbert, 2010).

Un aspecto interesante sobre el proceso de crecimiento y maduración de los oocitos (de ovario y órgano de Bidder) es que puede ser gatillado experimentalmente. Diferentes 
hormonas, gonadotropina coriónica humana (hCG), progesterona y testosterona, y los extractos de pituitaria, han sido utilizadas para inducir crecimiento, maduración y ovulación en varias especies de anfibios (Bravo et al., 1978). En el órgano de Bidder el uso de hormonas ha promovido diversos cambios dependiendo de la hormona que se ha utilizado, la especie que ha sido inducida y la etapa del desarrollo en la que esta se encuentra. En machos adultos de Bufo woodhousii, por ejemplo, las hormonas gonadotropina coriónica humana y gonadotropina de suero de yegua preñada provocaron un incremento en el tamaño de dicho órgano, así como también un incremento en el tamaño de los oocitos preexistentes (Pancak-Roessler y Norris, 1991). De igual manera, la exposición a hormonas tanto masculinas como femeninas en larvas de la especie Bufo vulgaris promovió un retraso en el desarrollo del OB en los individuos que fueron inducidos con testosterona y extractos de testículo y, dependiendo de la dosis, se observó un incremento de tamaño en el OB en las larvas expuestas a estradiol y extractos de pituitaria (Takahashi, 1956).

Otros compuestos químicos también pueden provocar crecimiento e incluso completa feminización en machos de distintas especies de anfibios, ya que actúan como disruptores endócrinos modificando la señal hormonal del individuo y alterando el sistema masculino reproductivo (Diamanti-Kandarakis et al., 2009). En los machos la feminización (inducción o desarrollo anormal de caracteres femeninos) generalmente se produce debido a desórdenes genéticos o adquiridos del sistema endócrino (Norris y Lopez, 2011; Blood et al., 2007). La exposición a disruptores endócrinos altera el señalamiento hormonal normal durante el desarrollo embriónico, cambiando permanentemente la morfología y función del sistema reproductivo en el adulto y su comportamiento reproductivo (McCoy et al., 2008). Varios pesticidas utilizados en la industria agrícola son disruptores endócrinos y cau- san anormalidades reproductivas en los individuos expuestos a ellos. La atrazina, uno de los pesticidas más usados en la industria agrícola alrededor del mundo, es conocida por ser un potente disruptor endócrino a bajas concentraciones. En anfibios, este compuesto suele ser más potente debido a que su actividad dentro de estos organismos se produce a niveles mucho más bajos en comparación con otros taxa (Hayes et al., 2002). Por ejemplo, Hayes et al., 2010, muestra las consecuencias reproductivas de la exposición de larvas de Xenopus laevis a la atrazina; ya que los machos adultos que fueron expuestos a la atrazina, desde la etapa larval hasta la metamorfosis, mostraron una desmasculinización (castración química) y completa feminización; es decir que el hermafroditismo observado en la metamorfosis resultó, en la mayoría de los casos, en una feminización completa y persistente ya que a pesar de ser genotípicamente machos los individuos de esta especie presentaban características fenotípicas femeninas, así como también eran reproductivamente funcionales y capaces de producir huevos viables.

Basados en estos conceptos, el presente estudio explora la inducción del crecimiento de los oocitos biderianos en bufónidos machos de la especie Rhinella marina, mediante la aplicación de distintos tratamientos hormonales y químicos con el fin de obtener oocitos de estadios oogénicos posteriores; por lo que el objetivo de este estudio fue acelerar el proceso de crecimiento en los oocitos del órgano de Bidder en machos adultos orquidectomizados de la especie Rhinella marina mediante el uso de hormonas y disruptores endócrinos.

\section{MATERIALES Y MÉTODOS}

Recolección y aclimatación.- Veinte y dos machos adultos de la especie Rhinella marina fueron colectados en las localidades de Jama, Manabí y Santo Domingo, Santo Domingo de los Tsáchilas a lo largo del año 2010. En el Laboratorio de Biología del Desarrollo y Biología Molecular (PUCE) estos 
animales fueron pesados, medidos (longitud boca-ano), fotografiados (no se muestran fotografías), identificados y distribuidos en cuatro terrarios (correspondientes al grupo control y a los tres tratamientos), en grupos de seis animales por cada terrario para, posteriormente, ser sometidos a un proceso de aclimatación a las condiciones estándares del laboratorio durante un período de dos meses.

Orquidectomía.- Finalizada la aclimatación, se removieron ambos testículos (oquidectomía bilateral) en los 22 machos adultos colectados. Los animales fueron anestesiados sumergiéndolos parcialmente en el anestésico MS-222 SIGMA-ALDRICH(Cat. No. E10521) por 20 minutos aproximadamente; a continuación, con tijeras de punta fina (MEDITEC cc), se realizó una incisión (de $15 \mathrm{~mm}$ de longitud aproximadamente) a $1 \mathrm{~cm}$ de la línea media del animal y a $30 \mathrm{~mm}$ de la base del abdomen y se removieron los testículos. En algunos casos la remoción fue parcial debido a que el órgano de Bidder (OB) rodeaba la punta del testículo; no obstante, se extrajo la mayor cantidad posible de testículo, evitando dañar el OB. Durante la operación se tomaron fotos y medidas de ambos OB en cada individuo. De igual manera, se tomaron fotos y medidas de los dos testículos removidos. Finalmente, las incisiones fueron suturadas con hilo quirúrgico reabsorberte Vicryl Plus antibacterial \# 3 ETHICON. Posterior a la operación, los animales fueron trasladados a un terrario individual, durante una semana, para permitir su recuperación.

Tratamientos (grupos experimentales).- Los sapos orquidectomizados fueron sometidos a cuatro tratamientos distintos durante un periodo de dos meses. Tanto las inyecciones, colocadas en la región intraperitoneal (Browne et al., 2006), como la solución que contenía el disruptor endocrino fueron administradas diariamente.

En el tratamiento "control: orquidectomía" se inyectó $100 \mu \mathrm{l}$ de Solución de Ringer para anfibios. Para el tratamiento "orquidec- tomía y progesterona" los animales recibieron una inyección intraperitoneal de solución de progesterona (SIGMA-ALDRICH, Cat. No.P8783) $7 \mu \mathrm{g} / \mathrm{ml}$ en solución Ringer.

En el tratamiento "orquidectomía y atrazina" los animales fueron expuestos a una solución de atrazina (Gesaprim 9WG ECUAQUIMICA) $0.1 \mathrm{ppb}$ en agua reposada, la cual se colocó en la base del terrario para que esté en contacto permanente con la superficie ventral del animal; para ello se realizó una previa aclimatación en la cual los animales fueron colocados individualmente en terrarios con 100 $\mathrm{ml}$ de agua reposada (sin disruptor endócrino) durante un periodo de dos semanas. Finalmente, para el cuarto tratamiento, "orquidectomía y atrazina + progesterona", los animales fueron sometidos tanto a las inyecciones de progesterona como a la exposición ventral de la solución de atrazina.

Cortes y análisis morfológico del OB.Finalizados los dos meses, los individuos de los cuatro tratamientos fueron sacrificados para extraer los órganos de Bidder izquierdo y derecho. De igual manera, se extrajo una porción de ovario de una hembra de la misma especie para comparar la morfología de ambas estructuras (OB y ovario). La morfología externa y el tamaño de los oocitos de dichos órganos fueron registrados por medio de micrografías utilizando un estereoscopio Stemi SV11 ZEISS y una cámara digital Canon PowerShot G9. A continuación, se fijó ambos órganos en Smith's durante 24 horas a temperatura ambiente. Después de este periodo, los órganos fueron sometidos a lavados seriales con agua destilada y fueron almacenados a temperatura ambiente en una solución de formaldehído $1.5 \%$ para su posterior análisis morfológico mediante cortes histológicos.

Para los cortes histológicos se modificó el protocolo descrito por Liu, 2006, para la tinción histoquímica de oocitos ya que se aumentó el tiempo de deshidratación y el 
de infiltracióncon paraplast (Histosec Pastillas-MERCK). Utilizando un micrótomo (820 Reichet HistoSTAT Rotary microtome) se cortó el tejido en secciones de $25 \mu \mathrm{m}$ de espesor, las cuales fueron colocadas en placas Superfrost ${ }^{\circledR}$ Plus FISHER SCIENTIFIC (Cat. No. 12-550-14). Para la tinción de las placas se siguió el protocolo de tinción con eosina y hematoxilina de Mayer (Lee, 1960) con algunas modificaciones en el tiempo de exposición a las soluciones.

Anexo 1. Tabla de la oogénesis de Rhinella marina. La oogénesis de $R$. marina fue clasificada en seis estadios definidos de acuerdo a la tabla de oogénesis de Dumont (1972) para Xenopus laevis (Brown et al., 2002).
Para establecer el estadio de la oogénesis tanto de las micrografías como de los cortes del OB de los machos orquidectomizados sometidos a los tratamientos, se midió el diámetro de los oocitos más grandes de cada órgano de Bidder y se lo comparó con la tabla de oogénesis descrita por Brown et al., 2002, para la especie Rhinella marina (Anexo 1).

Análisis estadístico.- Para observar el efecto de los tratamientos sobre el crecimiento de los oocitos del OB de los machos orquidec-

\begin{tabular}{|c|c|c|}
\hline Estadio & $\begin{array}{l}\text { Diámetro del } \\
\text { oocito }(\mu \mathrm{m})\end{array}$ & Características citológicas \\
\hline 1 & $50-300$ & $\begin{array}{l}\text { Citoplasma translúcido sin plaquetas de yema. Vs con } \\
\text { nucléolos y localizada centralmente. }\end{array}$ \\
\hline 2 & $300-450$ & $\begin{array}{l}\text { Citoplasma blanco-translúcido, debido a la formación de } \\
\text { plaquetas de yema. En la Vs se observan cromosomas } \\
\text { plumosos y varios nucléolos localizados principalmente } \\
\text { en la periferia. }\end{array}$ \\
\hline 3 & $450-600$ & $\begin{array}{l}\text { Oocito opaco y ligeramente pigmentado. Plaquetas de yema } \\
\text { de mayor longitud. El centralmente localizado núcleo contiene } \\
\text { nucléolos localizados principalmente en la periferia. } \\
\text { Se observa vascularización alrededor de los oocitos. }\end{array}$ \\
\hline 4 & $600-1000$ & $\begin{array}{l}\text { Pigmentación oscura presente en todo el oocito, con una } \\
\text { mayor concentración en el polo animal. Plaquetas de yem } \\
\text { mucho más largas y abundantes. Vs ligeramente desplazad } \\
\text { hacia el polo animal. Numerosos nucléolos localizados en la } \\
\text { periferia del núcleo. Visible vascularización alrededor de los } \\
\text { oocitos. }\end{array}$ \\
\hline 5 & $1000-1200$ & $\begin{array}{l}\text { Polos animal y vegetal claramente visibles en el oocito con } \\
\text { una mayor concentración de pigmento en el polo animal. } \\
\text { Plaquetas de yema mucho más largas y abundantes. } \\
\text { La Vs es desplazada hacia la región animal. Numerosos } \\
\text { nucléolos localizados en la periferia del núcleo. No se observa } \\
\text { RVs. vascularización alrededor de los oocitos. }\end{array}$ \\
\hline 6 & $1200-1400$ & $\begin{array}{l}\text { Polos animal y vegetal claramente visibles en el oocito, con } \\
\text { una mayor concentración en el polo animal. No se distingue la línea } \\
\text { ecuatorial de pigmento en el ligeramente pigmentado polo vegetal. } \\
\text { Se observa vascularización alrededor de los oocitos. En los oocitos } \\
\text { de este estadio no se analizó la RVs y la histología. }\end{array}$ \\
\hline
\end{tabular}

* Los estadios de la oogénesis fueron definidos de acuerdo a Xenopus laevis (Dumont 1972). Vs, vesícula germinal; RVs, ruptura de la vesícula germinal 
tomizados se realizó un Análisis de Varianza (ANOVA) factorial ( $4 \times 2$ ), utilizando como variable el diámetro máximo de los oocitos del $\mathrm{OB}$ derecho e izquierdo de los animales de cada tratamiento. También se realizaron comparaciones ortogonales para determinar la combinación de los tratamientos frente al control o entre tratamientos del órgano de Bidder de cada lado en el programa estadístico SPSS 17.0. Dichas interacciones fueron graficadas mediante diagramas de caja (box plots) utilizando el programa Golden Grapher 7.0.

\section{RESULTADOS}

Descripción morfológica del ovario de Rhinella marina.- La porción de ovario extraído a la hembra de $R$. marina contenía ooci- opaco y transparente), diámetros y diferentes características morfológicas y citológicas. Los oocitos de todos los estadios se encontraban rodeados por una capa de células foliculares.

Descripción de la gónada masculina de los machos de Rhinella marina.- La gónada de los machos, durante la orquidectomización, presentó las dos estructuras que forman parte de la gónada masculina en bufónidos; el órgano de Bidder (OB) y el testículo. Ambas estructuras presentaron diferencias en su morfología, claramente observables en la zona de transición entre el testículo y el OB (Figura 1A-B).
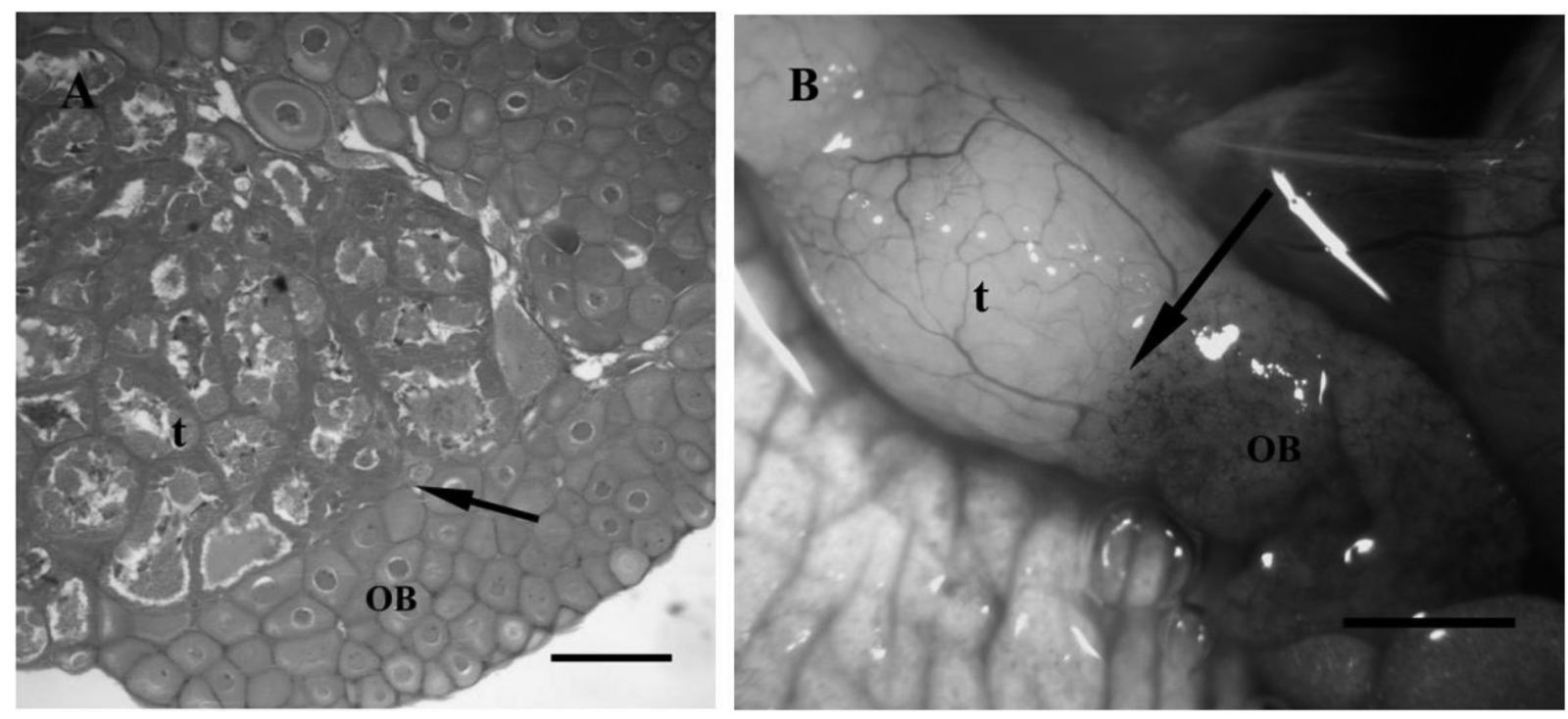

Figura 1. Zona de transición testículo-órgano de Bidder. (A) Corte histológico del órgano de Bidder izquierdo, animal \# 12 del tratamiento orquidectomía y progesterona. (B) Micrografía del órgano de Bidder izquierdo, animal \# 10 previo al tratamiento orquidectomía y progesterona. Las flechas indican la zona de transición. La barra representa $500 \mu \mathrm{m}$ (A) y $2 \mathrm{~mm}$ (B). t, testículo; OB, órgano de Bidder.

tos en diferentes estadios de la oogénesis. Los oocitos más grandes (1 $200 \mu \mathrm{m}$ de diámetro) presentaron una clara diferenciación entre el polo animal y vegetal con una mayor concentración de pigmento (de color café oscuro) en el polo animal; la vesícula germinal se encontraba desplazada hacia este polo. También se encontraron oocitos de menor tamaño que presentaban coloraciones (café claro, blanco
Los testículos removidos, observados in vivo, eran de color amarillo pálido, forma ovalada alargada, con alta vascularización y un ensanchamiento en la región de unión al OB. En los cortes histológicos se observaron túbulos seminíferos con espermatogonias, espermatocitos y agrupaciones de espermatozoides. Los testículos izquierdo y derecho de los 22 animales midieron en promedio $21.3 \mathrm{~mm}$ de largo y $4 \mathrm{~mm}$ de ancho y pesaron $106 \mathrm{mg}$. 
Durante las operaciones, se encontró al OB adherido al extremo anterior de cada testículo y unido a un cuerpo graso. Estructuralmente, presentó una coloración rosada-pálida y un tamaño y forma variables. En algunos animales se observaron manchas de color negro (células con melanina) alrededor de todo el OB o en determinadas regiones. Las mediciones del diámetro de los oocitos biderianos de mayor tamaño de los OB de los veinte y dos machos arrojaron un diámetro promedio de $189 \mu \mathrm{m}$. La mayoría de estos oocitos, según la tabla de oogénesis para $R$. marina (Brown et al., 2002), se encontraban en el estadio I de la oogénesis mientras que únicamente tres de los veinticuatro animales presentaron oocitos de estadio II. Morfológicamente, los oocitos biderianos eran ligeramente transparentes con una gran vesícula germinal fácilmente visible in vivo.

Efecto de la orquidectomía y la administración de progesterona y atrazina sobre el OB.- Después de los dos meses de tratamiento se observó una mayor vascularización en el órgano de Bidder y los oocitos de todos los tratamientos y un considerable aumento de tamaño de dichos oocitos, alcanzado un diámetro promedio general de $346 \mu \mathrm{m}$ (Tabla 1 aquí). En los cortes histológicos también se pudieron observar algunas zonas con oocitos atrésicos y ciertas zonas con manchas de color café o negro, correspondientes a células con melanina.

Al comparar todos los tratamientos, mediante ANOVA factorial ( 4 x 2 ) de los datos transformados (Coeficiente Variación datos no transformados $=58.6 \%$ ) se encontró diferencias altamente significativas entre los tratamientos $(\mathrm{F}=4.3 ; \mathrm{p}=0.009)$ pero no entre órgano de Bidder izquierdo y derecho $(\mathrm{F}=$ $1.4 ; \mathrm{p}>0.05)$.

En las comparaciones ortogonales, se encontró un media mayor, numéricamente, en el tratamiento "control: orquidectomía" del OB izquierdo comparado con los otros tratamien- tos; sin embargo, no se encontraron diferencias estadísticas $(\mathrm{p}=0.09)$. De igual manera, se observó una diferencia numérica mayor entre el tratamiento progesterona frente al control pero tampoco se encontraron diferencias significativas $(p=0.07)$. Entre los demás tratamientos no se observaron diferencias significativas.

En cuanto al OB derecho, se realizó una nueva comparación ortogonal con los datos transformados (Coeficiente Variación datos no transformados $=63,6 \%$ ), la cual arrojó diferencias significativas entre todos los tratamientos $(p=0.04)$, entre el control y los tratamientos $(\mathrm{p}=0.02)$, entre el tratamiento progesterona frente a los demás tratamientos y el control $(p=0.016)$ y entre el tratamiento progesterona frente al tratamiento atrazina $(p=0.05)$, y diferencias altamente significativas entre el tratamiento progesterona frente al control $(\mathrm{p}=0.007)$.

En los diagramas de caja se observó que existían datos atípicos de oocitos que se encontraban muy por encima o debajo de la mediana, también se pudo comprobar la variabilidad de medianas entre los tratamientos de los órganos de Bidder izquierdo y derecho (Figura 2A-B). En el OB derecho, tanto el tratamiento progesterona como el de atrazina con progesterona presentaron datos atípicos muy por encima de su mediana.

Orquidectomía y administración de solución salina.- En los animales pertenecientes al tratamiento "control: orquidectomía", se observó un ligero incremento en el diámetro de los oocitos, así como también en su número. En los cortes histológicos, las micrografías y el diámetro máximo de los oocitos se encontró un gran número de los oocitos en estadio I de la oogénesis (Figura 3A-B). Para el OB derecho, el promedio del diámetro máximo de los oocitos fue de $207 \mu \mathrm{m}$ y del órgano izquierdo fue de $229 \mu \mathrm{m}$. Dentro de este tratamiento, el animal \# 1, presentó una morfología distinta en su órgano de Bidder; antes de la operación 
A)

\section{ÓRGANO DE BIDDER IZQUIERDO}

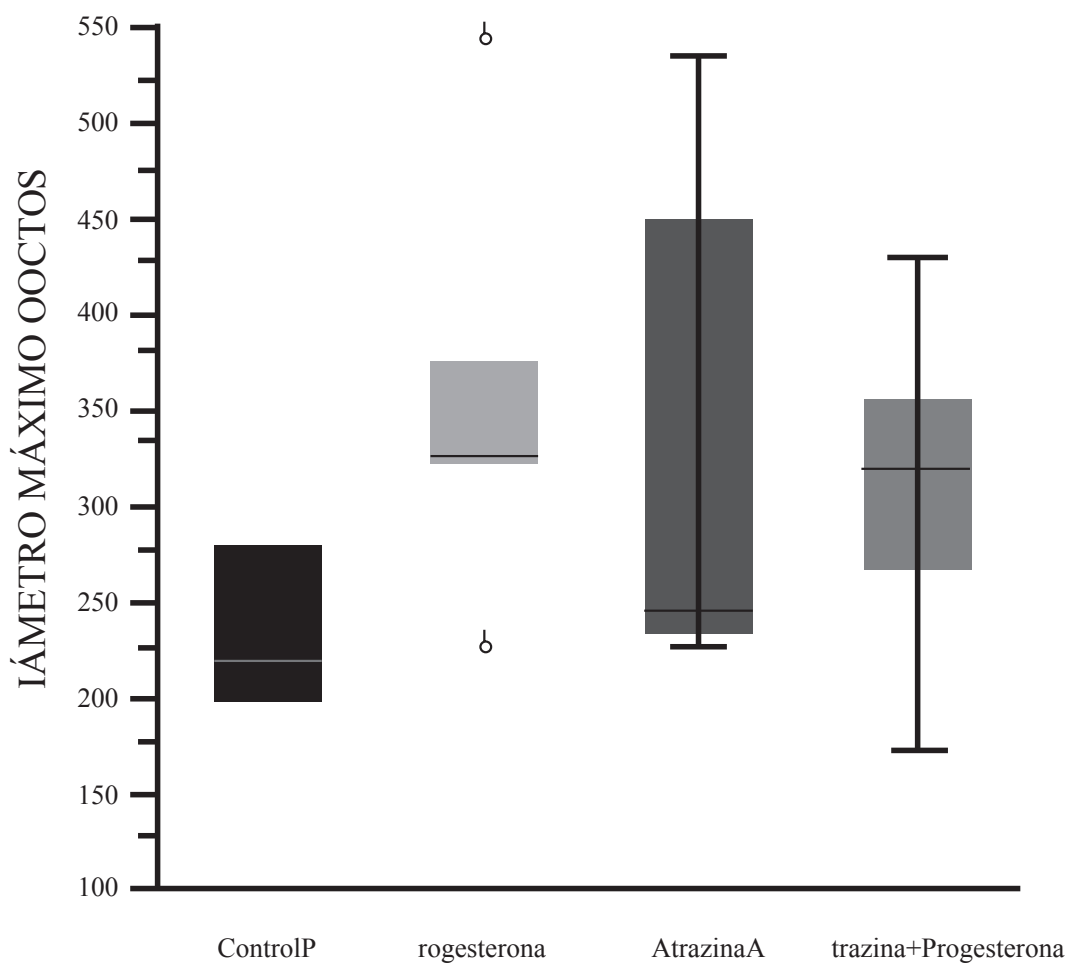

B)

\section{ÓRGANO DE BIDDER DERECHO}

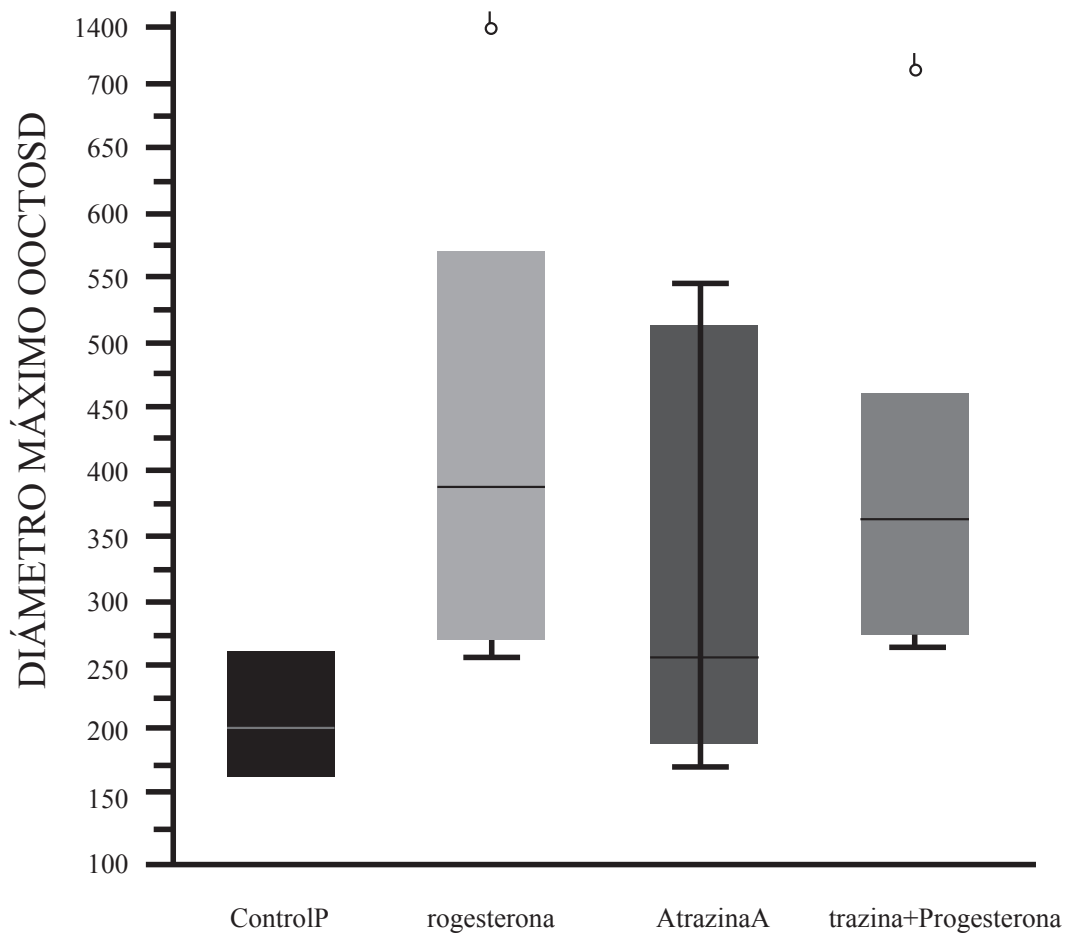

Figura 2. Diagramas de caja del diámetro máximo de los oocitos de los órganos derecho e izquierdo de los machos orquidectomizados. 
se pudo observar que tanto el órgano izquierdo como el derecho poseían una coloración café oscura (atípica) alrededor de todo el OB y que dichos órganos eran bastante pequeños.
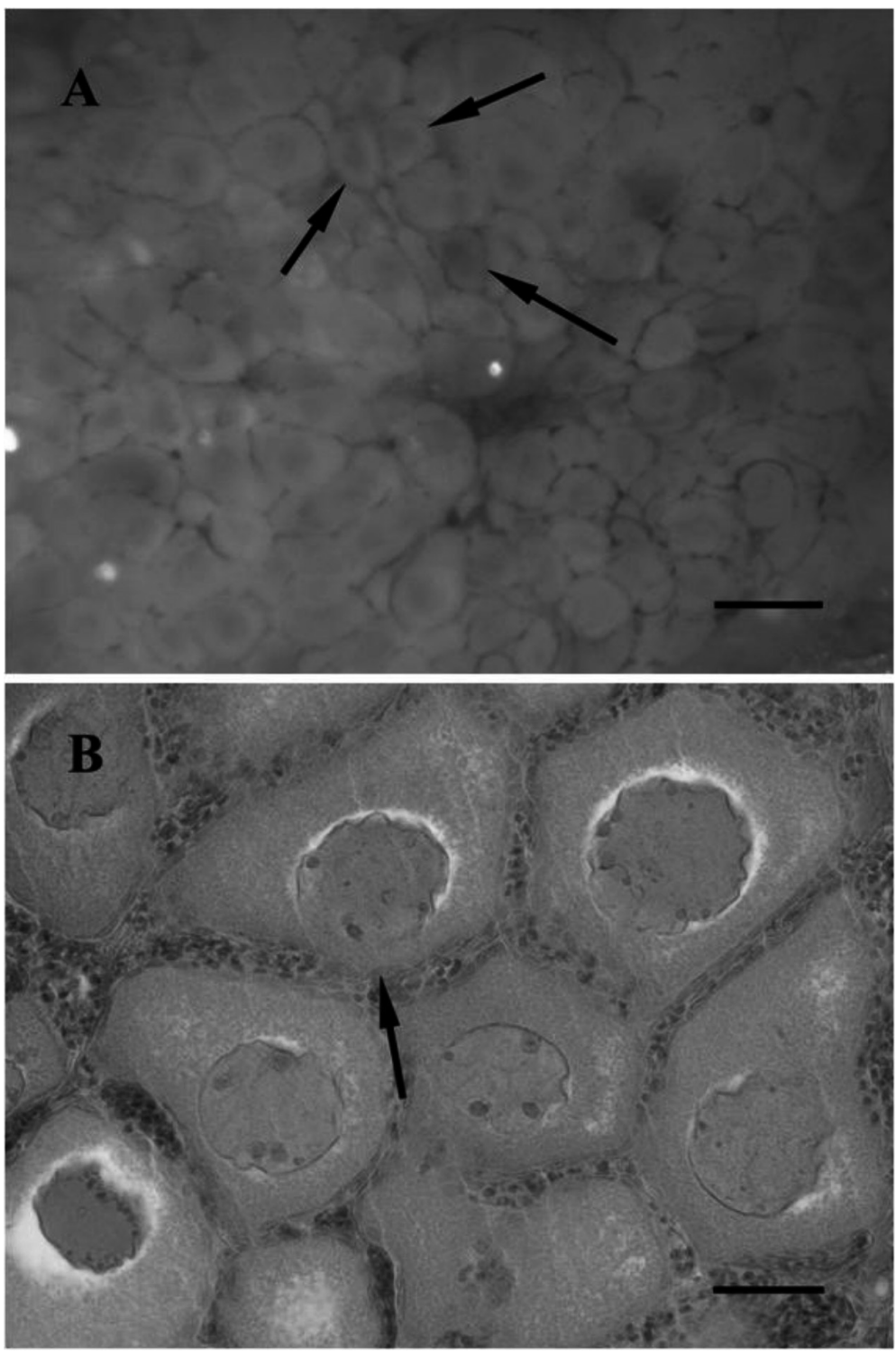

Figura 3. Oocitos tratamiento control: orquidectomía. (A) oocitos macho control \# 6, órgano de Bidder derecho. Las flechas señalan oocitos en estadio I de la oogénesis; transparentes, vesícula germinal visible. (B) oocitos macho control \# 2, órgano de Bidder derecho. La flecha indica un oocito estadio I, núcleo con numerosos nucléolos distribuidos aleatoriamente. La barra representa $250 \mu \mathrm{m}$ (A) y $60 \mu \mathrm{m}(\mathrm{B})$.

Posterior al tratamiento, se observó un notable crecimiento en ambos órganos y una mayor proliferación de oocitos.
Orquidectomía y administración de progesterona.- En el tratamiento "orquidectomía y progesterona" se encontraron oocitos de estadio I, II y III (Figura 4A- B- C- D). De los cuales su gran mayoría pertenecían al estadio II de la oogénesis. El promedio del diámetro máximo de los oocitos del OB derecho fue de $546 \mu \mathrm{m}$ y del órgano izquierdo fue de $352 \mu \mathrm{m}$. Dentro de este tratamiento, tres animales presentaron oocitos pigmentados pertenecientes al estadio III o IV de la oogénesis. En el animal \# 12 se encontró el oocito de mayor tamaño registrado en todos los tratamientos (1 $400 \mu \mathrm{m})$, en el cual se observó la morfología característica de un oocito perteneciente al estadio VI de la oogénesis (Figura 5A-B).

Orquidectomía y administración de atrazina.- En el tratamiento "orquidectomía y atrazina" se encontraron oocitos pertenecientes a los estadios I, II y III de la oogénesis. En las mediciones del diámetro máximo de los oocitos también se observaron diámetros pertenecientes a estos tres estadios arrojando un diámetro promedio para el órgano de Bidder derecho de $323 \mu \mathrm{m}$ y para el izquierdo de $322 \mu \mathrm{m}$.

\section{Orquidectomía y adminis-} tración de atrazina + progesterona.- En el tratamiento "orquidectomía y atrazina + progesterona" las micrografías y los cortes histológicos muestran oocitos de estadios I, II, III y IV de la oogénesis; la mayoría de los cuales pertenecían al estadio II de la oogénesis. El diámetro promedio del $\mathrm{OB}$ derecho fue de $405 \mu \mathrm{m}$ y el izquierdo fue de $310 \mu \mathrm{m}$. 


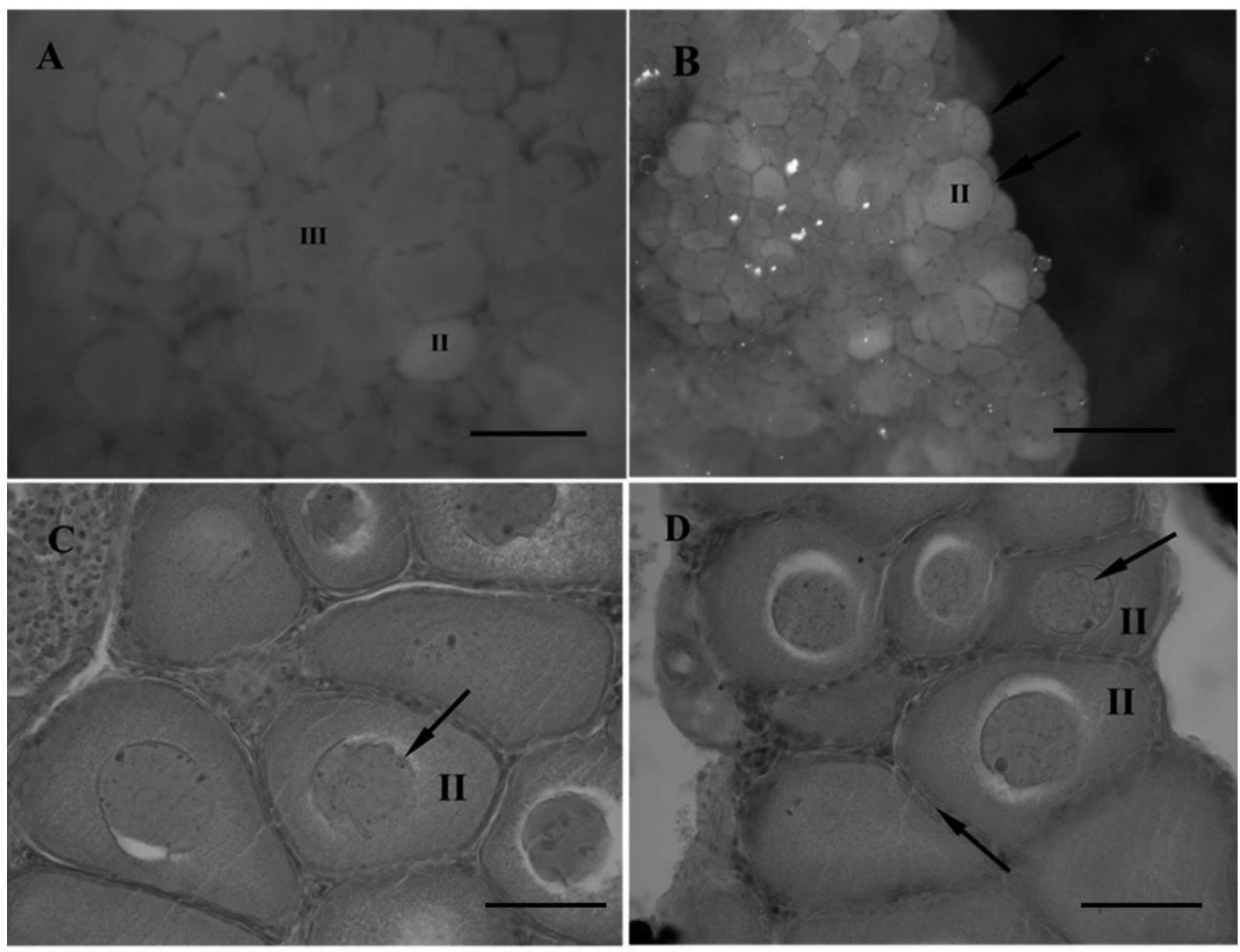

Figura 4. Oocitos tratamiento orquidectomía y progesterona. (A) oocitos del órgano de Bidder izquierdo del macho \# 9. (B) oocitos del órgano de Bidder derecho del macho \# 10. Las flechas señalan oocitos estadio II altamente vascularizados. (C) oocitos órgano de Bidder izquierdo macho \# 7. La flecha indica un oocito de estadio II con la vesícula germinal con numerosos núcleos ubicados en la periferia. (D) oocitos órgano de Bidder derecho individuo \# 10. Flecha superior señala cromosomas dentro del núcleo, flecha inferior, pared de células foliculares. Los números romanos indican el estadio de la oogénesis de los oocitos. La barra representa $200 \mu \mathrm{m}(\mathrm{A}) ; 700 \mu \mathrm{m}$ (B); $90 \mu \mathrm{m}(\mathrm{D}$ y C).

\section{DISCUSIÓN}

Órgano de Bidder, ovario y testículos de Rhinella marina.- Similar al ovario de Xenopus laevis, el ovario de una hembra adulta de Rhinella marina posee oocitos mononucleados de diferentes estadios vitelogénicos rodeados de células foliculares. Estos oocitos, en cada estadio, presentan características citológicas y morfológicas únicas y distinguibles entre sí (Brown et al., 2002; Dumont, 1978). Brown et al., 2002, describe dichas características en la tabla de oogénesis para $R$. marina, la cual fue utilizada para determinar el estadio de la oogénesis en la que se encontraban los oocitos biderianos de cada tratamiento, que también fueron comparados con los oocitos de la sección de ovario extraído de la hembra. De igual manera, mediante los cortes de los testículos removidos, se pudo establecer que los machos orquidectomizados estaban sexualmente maduros ya que presentaron densas concentraciones de espermatozoides y espermátidas dentro de los túbulos seminíferos (Zug y Zug, 1979)

La similaridad morfológica de los oocitos biderianos, estructuralmente equivalentes a los oocitos previtelogénicos del ovario ( $\mathrm{Fa}-$ rias et al., 2002), con los oocitos del ovario 


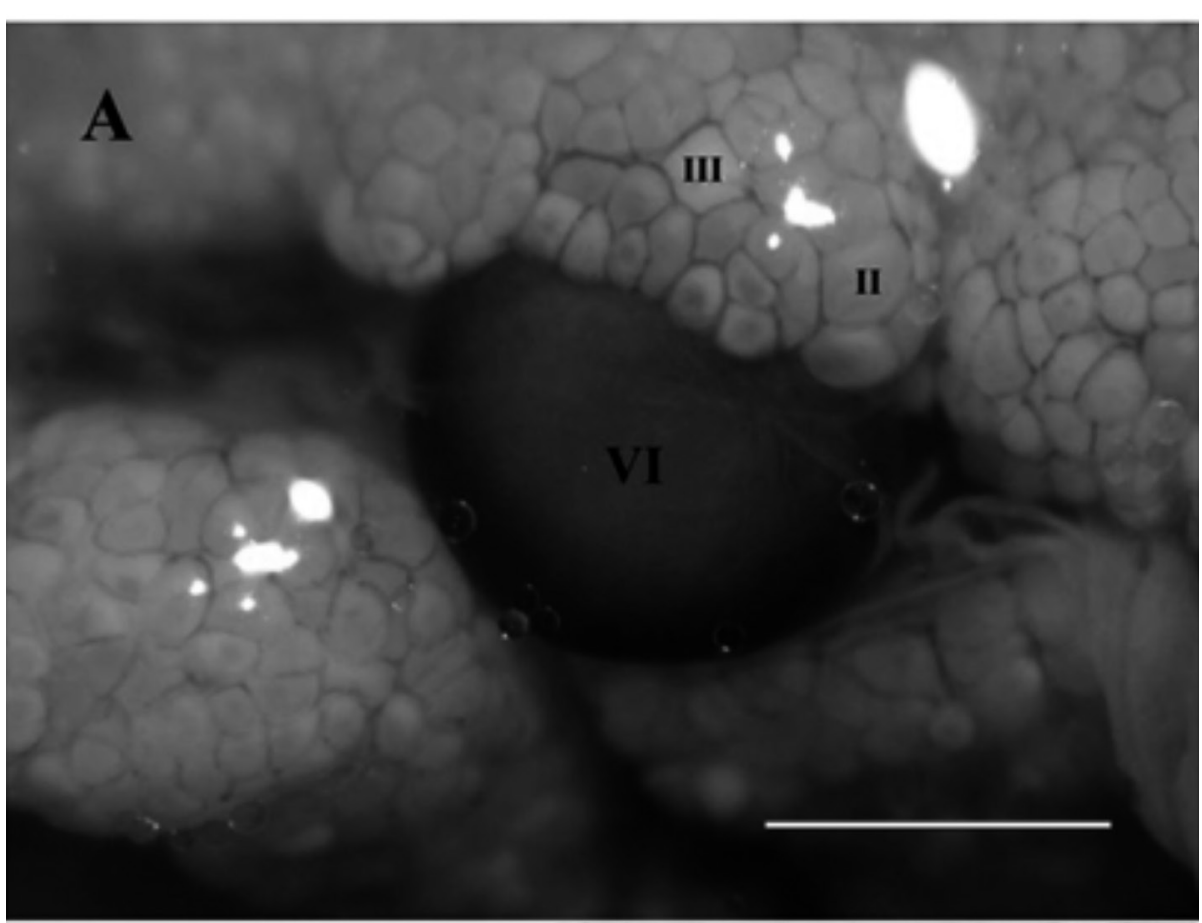

algunos individuos en cada tratamiento, es una característica común dentro de este órgano ya que ha sido encontrada en algunos estudios del OB (Farias et al., 2002; Pancak-Roessler y Norris, 1991). En los machos orquidectomizados de todos los tratamientos, excepto el control, también se pudieron encontrar pequeños gránulos de color café (células con melanina) localizados en la periferia del ooplasma de los oocitos biderianos, equivalente a lo hallado por Pancak-Roessler y Norris, 1991.

Efecto de la orquidectomía $\mathbf{y}$ la administración de progesterona $y$ atrazina sobre el OB.- La aplicación de progesterona $y$ atrazina exógenas

Figura 5. Oocitos estadio II, III y VI órgano de Bidder derecho animal \# 12 tratamiento orquidectomía y administración de progesterona. (A) Micrografía. (B) Corte histológico. Los números romanos corresponden al estadio de la oogénesis en el que se encuentran los oocitos. t, testículo; Vs, vesícula germinal. La barra representa $1 \mathrm{~mm}$ (A y B).

de la hembra permitió la determinación del estadio de la oogénesis de los oocitos biderianos en los machos orquidectomizados de este estudio. La presencia de oocitos atrésicos, observada en ciertas regiones del OB de en los machos orquidectomizados de Rhinella marina provocó el crecimiento de los oocitos del órgano de Bidder, claramente observable en el promedio de los oocitos biderianos de mayor tamaño de todos los tratamientos durante la operación y posterior a los tratamientos (de 189 a $346 \mu \mathrm{m}$ ). 

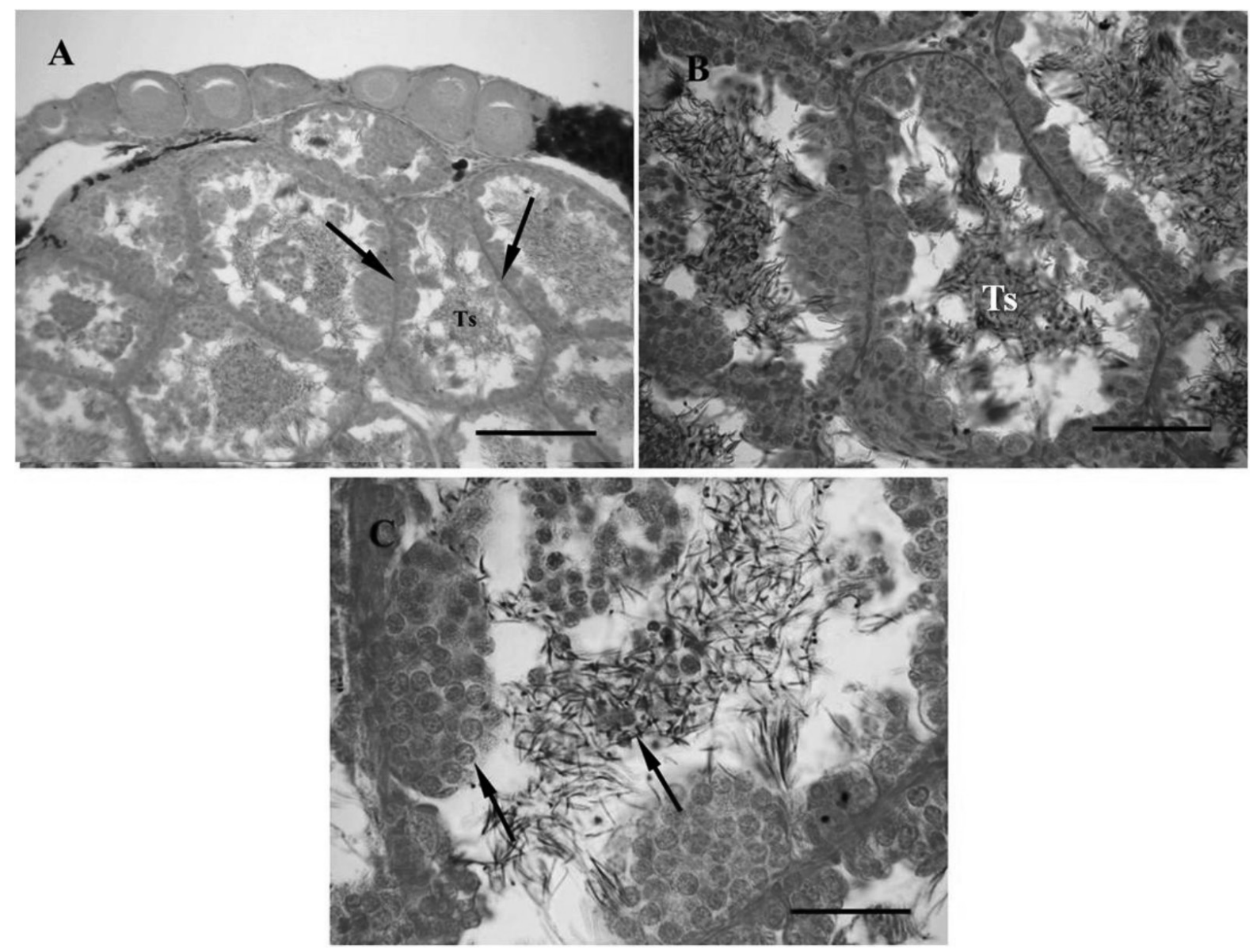

Figura 6. Cortes histológicos del testículo de Rhinella marina, testículo derecho animal \# 1 tratamiento control: orquidectomía. (A) Testículo rodeado de oocitos pertenecientes al órgano de Bidder. Las flechas señalan un túbulo seminífero; Ts. (B) Túbulo seminífero; Ts. (C) espermatozoides de R. marina. Flecha izquierda, espermatocito; flecha derecha, espermatozoides. La barra representa $200 \mu \mathrm{m}$ (A); $100 \mu \mathrm{m}$ (B); $50 \mu \mathrm{m}$ (C).

De igual manera, las diferencias significativas en el ANOVA factorial y las comparaciones ortogonales indican que la tasa de crecimiento fue distinta en cada tratamiento aplicado; ya que se encontraron diferencias al realizar la comparación entre los órganos de Bidder de ambos lados de los individuos "control: orquidectomía" frente a los individuos de los demás tratamientos. Sin embargo, en el lado izquierdo el ANOVA arrojó diferencias numéricamente mayores y no estadísticamente significativas mientras que para el lado derecho estas diferencias sí fueron estadísticamente significativas; esto pudo deberse a que en el lado derecho se registraron los oocitos de mayor diámetro en los tratamientos "orquidectomía y progesterona" y "orquidectomía y atrazina + progesterona".
Las diferencias también se observaron al analizar las medias de cada tratamiento; la media más alta perteneció al tratamiento "orquidectomía y progesterona" (483 $\mu \mathrm{m})$, seguida por el tratamiento "orquidectomía y atrazina + progesterona" $(358 \mu \mathrm{m})$, el tratamiento "orquidectomía y atrazina" $(322 \mu \mathrm{m})$ y finalmente el tratamiento "orquidectomía: control", con una media de $218 \mu \mathrm{m}$; lo que indica que cada compuesto actúa de forma distinta en el proceso de crecimiento de los oocitos biderianos y que la hormona progesterona fue más exitosa que el disruptor endócrino atrazina en la inducción de crecimiento; esto también pudo comprobarse en las comparaciones ortogonales en las que se registraron diferencias significativas entre el tratamiento "orquidectomía y progesterona" frente al tratamiento "orquidectomía y atrazina". 

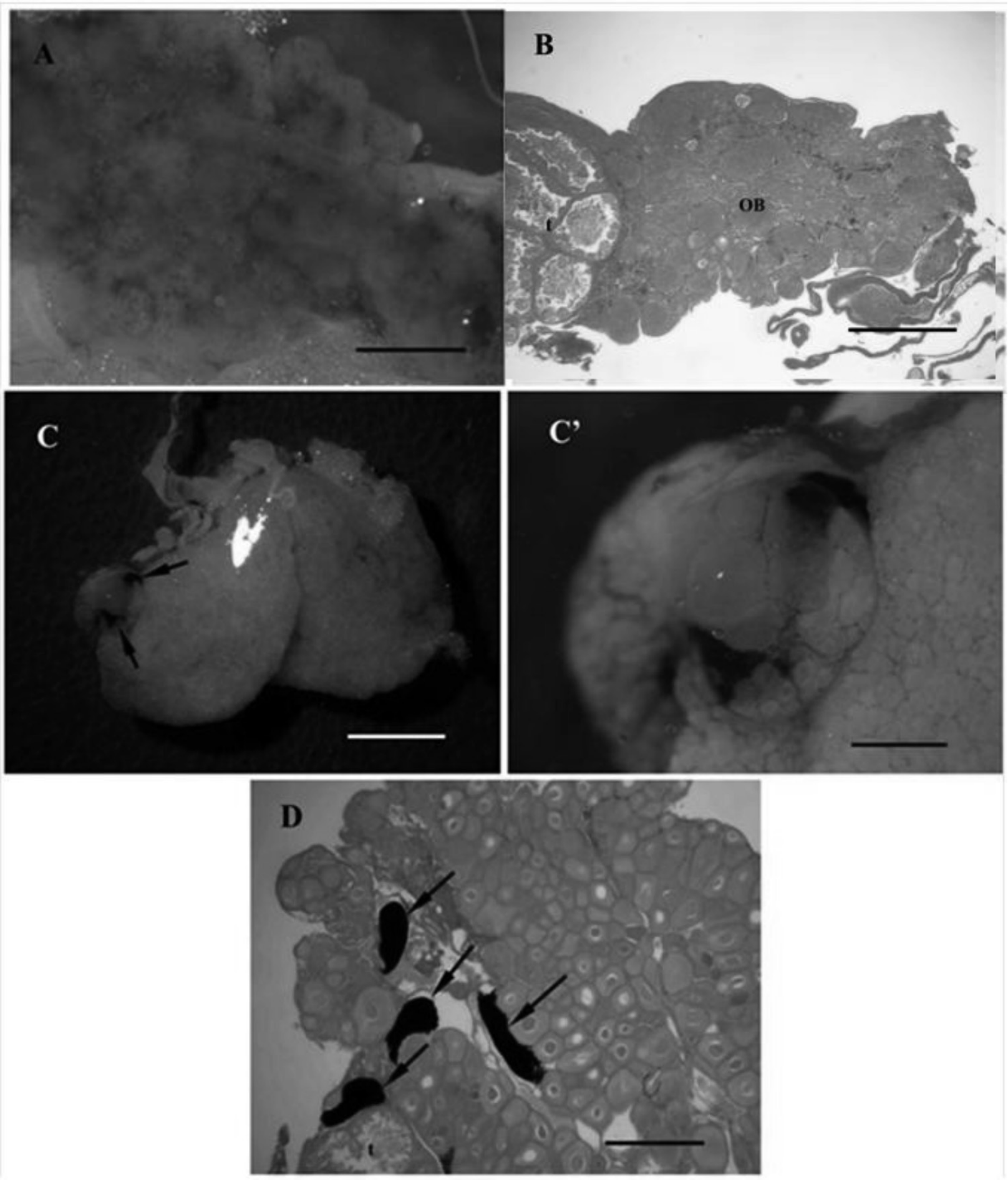

Figura 7. Órganos de Bidder con zonas de oocitos atrésicos y zonas manchadas. (A) Micrografía órgano de Bidder derecho animal \# 3 tratamiento orquidectomía y atrazina. Se observan manchas alrededor de toda la estructura. (B) Corte histológico órgano de Bidder derecho animal \#3, con grades zonas de oocitos atrésicos. (C) Micrografía órgano de Bidder izquierdo animal \# 8 después del tratamiento orquidectomía y administración de progesterona. (C') Manchas encontradas previo y después del tratamiento. (D) Corte histológico órgano de Bidder izquierdo animal \# 8. Las flechas señalan las manchas halladas. $t$, testículo. OB, órgano de Bidder. Las barras representan $1 \mathrm{~mm}(\mathrm{~A}) ; 900 \mu \mathrm{m}(\mathrm{B}) ; 3 \mathrm{~mm}(\mathrm{C}) ; 800 \mu \mathrm{m}(\mathrm{D}) ; 700 \mu \mathrm{m}\left(\mathrm{C}^{\prime}\right)$. 
La remoción de los testículos sin la administración de hormonas y disruptores endócrinos (tratamiento control: orquidectomía) provoca el alargamiento del $\mathrm{OB}$ y el incremento en el tamaño de los oocitos biderianos (Brown et al., 2002; Pancak-Roessler y Norris, 1991). Al extraer los testículos, el OB crece y sus oocitos alcanzan estadios de la oogénesis superiores, ya que se elimina el efecto inhibitorio de los testículos sobre el crecimiento de este órgano (Falconi et al., 2007). Este efecto, se cree que es producido por ciertas hormonas masculinas secretadas por los testículos (Zaccanti y Tognato, 1976).

Todos los oocitos de los animales del tratamiento "control: orquidectomía", aumentaron de tamaño (218 $\mu \mathrm{m}$ diámetro máximo promedio) y todos ellos pertenecían al estadio I de la oogénesis después de los dos meses de administración de la solución salina (Brown et al., 2002) (Figura 3). Pancak-Roessler y Norris, 1991, en su estudio sobre el efecto de las hormonas gonadotropinas en el OB, obtuvieron resultados similares después de tratar a machos orquidectomizados con una solución salina durante un mes. La similaridad de resultados a diferentes tiempos puede ser explicada con el estudio de Brown et al., 2002, el cual concluye que la mayor parte del incremento en el diámetro de los oocitos se produce después del primer mes de realizada la orquidectomía.

Se conoce que los esteroides son las principales hormonas que están involucradas en la inducción de la diferenciación sexual. Varios estudios sobre la aplicación de estas hormonas en embriones y larvas de anfibios han demostrado que existe una gran variedad de esteroides que causan reversión sexual en anfibios (Norris y Lopez, 2011; Hayes, 1998). En este estudio, se utilizó progesterona para promover el crecimiento de los oocitos del órgano de Bidder ya que a pesar de que fue utilizada en machos adultos su acción dentro del organismo fue dirigida al $\mathrm{OB}$, el cual posee oocitos previtelogénicos (Falconi et al., 2007; Brown et al., 2002; Farias et al., 2002).

El mecanismo de acción observado de la progesterona está relacionado con el proceso de oogénesis. Los oocitos del OB en los machos adultos de $R$. marina se encuentran en estadios previtelogénicos de la oogénesis (estadios I y II); es decir que posiblemente han alcanzado el arresto en profase I pero que no han empezado a acumular plaquetas de yema (Gilbert, 2010; Falconi et al., 2007; Farias et al., 2002).

Tabla 1. Medidas promedio de los oocitos del órgano de Bidder de los machos orquidectomizados después de los dos meses de tratamiento. Entre paréntesis estadio de la oogénesis de los oocitos de máximo diámetro ( $\mathrm{I}=$ izquierdo; $\mathrm{D}=$ derecho).

TRATAMIENTO

DIAMETRO MÁXIMO OOCITOS

$(\mu \mathrm{m})$

I $\quad$ D

\begin{tabular}{ccc}
\hline Control & $228(1)$ & $207(1)$ \\
Progesterona & $352(2)$ & $532(3)$ \\
Atrazina & $322(2)$ & $324(2)$ \\
Progesterona y Atrazina & $437(2)$ & $406(2)$ \\
\hline PROMEDIO DE CADA & 310 & 382 \\
LADO & & \\
\hline PROMEDIO TOTAL & $\mathbf{3 4 6}$ \\
\hline
\end{tabular}


La vitelogénesis, se produce después de que los oocitos han alcanzado el estadio III de la oogénesis (400 $\mu \mathrm{m}$ de diámetro aproximadamente). Los oocitos del OB, por ende, lograrán estadios de la oogénesis mayores una vez que inicien el proceso de vitelogénesis (Gilbert, 2010; Schuetz, 1974). En este estudio, tanto la hormona progesterona como el disruptor endócrino atrazina fueron utilizados para inducir la iniciación de la vitelogénesis.

El análisis estadístico en este estudio confirmó que la hormona progesterona participó dentro del proceso de crecimiento, ya que el tratamiento progesterona resultó en oocitos con un diámetro máximo por encima de la media grupal y del propio tratamiento (oocitos de estadios IV y VI) (Figura 4). Las comparaciones ortogonales afirman lo dicho anteriormente ya que se encontró en los órganos de Bidder de los machos del tratamiento "orquidectomía y administración de progesterona" frente a los machos "control: orquidectomía" diferencias mayores en el lado izquierdo $(p=0.07)$ y diferencias altamente significativas en el lado derecho $(p=0.007)$.

Sin embargo, los datos atípicos, encontrados por encima y debajo de la mediana en el diagrama de caja del tratamiento progesterona, nos indican que este tratamiento fue parcialmente exitoso ya que la cantidad obtenida de oocitos crecidos fue muy baja (solo un oocito estadio VI y pocos estadio IV) (Figura 2A-B). Esto puede deberse a que las hormonas esteroides actúan de manera distinta en cada especie e incluso en cada individuo (Norris y Lopez, 2011).

Estudios con varios tipos de esteroides en diferentes especies de anfibios demuestran que existe una gran variabilidad de resultados (Wallace et al., 1999; Hayes, 1998), sugiriendo que el efecto hormonal puede variar entre especies e incluso de entre organismos. Dependiendo de la especie, esteroides exógenos pueden ser metabolizados a compuestos inactivos o compuestos más potentes. Los factores que producen dicha variabilidad pueden ser ambientales (temperatura, alimentación, tiempo y duración del tratamiento; entre otros) o intrínsecos del propio organismo (metabolismo) (Norris y Lopez, 2011). En animales jóvenes de Xenopus laevis, por ejemplo, se requiere un periodo de 1 a 1.5 años para producir oocitos completamente desarrollados, mientras que en hembras estimuladas con gonadotropinas solo se requieren dos meses (Hausen y Riebesell, 1991). También se ha determinado que la síntesis y fosforilación de nuevas cadenas de vitelogenina son eventos altamente relacionados y que se requiere aproximadamente dos horas (dependiendo del metabolismo del organismo) para la síntesis de una nueva molécula de vitelogenina. Esto sugiere que, dentro de este estudio, posiblemente los factores tiempo, alimentación o concentración hormonal estuvieron involucrados en la producción del bajo número de oocitos crecidos. No obstante, los resultados presentados aquí son significativos al ser comparados con otros estudios en los que se obtuvieron oocitos vitelogénicos que alcanzaron estadios III y IV en el proceso de oogénesis (Brown, 2002; Pancak-Roessler y Norris, 1991) (Figura 5).

Numerosos químicos fabricados por el hombre actúan dentro del metabolismo de los organismos imitando y alternado proceso fisiológicos estrógeno-dependientes. Estas sustancias químicas, llamadas disruptores endócrinos, producen alteraciones significativas e irreversibles en los procesos de reproducción y desarrollo a concentraciones extremadamente bajas. En este estudio se utilizó atrazina (un tipo de disruptor endócrino) para provocar una alteración irreversible en el ciclo hormonal de la especie Rhinella marina y obtener oocitos biderianos de estadios oogénicos superiores.

Entre otros efectos, la atrazina altera tejidos reproductivos masculinos en los organismos que han sido expuestos al químico durante el desarrollo, causando demasculinización y feminización en las gónadas de machos vertebrados (Hayes et al., 2011). En el tratamiento "orquidectomía y atrazina", la atrazina provocó el crecimiento de los oocitos biderianos de los 
machos orquidectmizados alcanzado hasta estadios II y III de la oogénesis. En el diagrama de caja, a pesar de que existen datos alejados de la mediana no se observan datos atípicos (Figura 2A-B). Igualmente, al comparar la media de este tratamiento $(323 \mu \mathrm{m})$, con el promedio del tratamiento progesterona $(484 \mu \mathrm{m})$, especialmente en el órgano de Bidder derecho, se encontraron diferencias significativas $(p=0.05)$, lo que indica que este tratamiento fue menos exitoso que el tratamiento "orquidectomía y progesterona" en el crecimiento de los oocitos biderianos.

Se cree que el mecanismo de acción de la atrazina dentro del organismo está relacionado con un incremento en la producción de la enzima aromatasa P450; la cual es responsable de convertir andrógenos en estrógenos (testosterona a estradiol). Como consecuencia de su incremento, se produce un aumento en los niveles de estrógeno en los machos de varias especies de anfibios y peces (grupos en los que este efecto ha sido ampliamente estudiado) lo que finalmente provoca la feminización de los individuos. Sin embargo, este mecanismo de acción no es concluyente en los anfibios ya que se ha observado que la aromatasa P450 puede ser activada mediante dos vías distintas en peces y mamíferos (Norris y Lopez, 2011). En este estudio se observaron los efectos feminizadores que provoca la conversión de andrógenos en estrógenos, ya que se observó un incremento de tamaño en los oocitos del OB de los machos; sin embargo, dichos efectos no fueron pronunciados ya que no se encontraron oocitos biderianos de estadios vitelogénicos tardíos.

La ausencia de oocitos biderianos de estadios vitelogénicos tardíos pudo deberse a que posiblemente la concentración de atrazina dentro del tratamiento no fue la adecuada (Hayes et al., 2003). De igual manera, el uso de la atrazina como disruptor endócrino nunca había sido probado en especies terrestres adultas, por lo que se tuvo que idear un mecanismo mediante el cual los individuos estuvieran en contacto permanente con el químico. Además del efecto feminizador en las gónadas masculinas, se conoce que la atrazina deteriora el sistema inmune aumentando la sensibilidad de los anfibios a infecciones (Norris y Lopez, 2011). La inmunodepresión, por tanto, pudo ser otro factor que influyó en la obtención de oocitos de estadios vitelogénicos tempranos, a pesar de que ninguno de los animales dentro de este tratamiento presentó enfermedades o infecciones.

Al observar, de manera individual, los resultados del tratamiento "orquidectomía progesterona", "orquidectomía: control" y "orquidectomía y atrazina." Se puede inferir que los resultados obtenidos dentro del tratamiento "orquidectomía y atrazina + progesterona" se deben en gran parte a la acción de la progesterona más que a la acción de la atrazina y que además ambos compuestos no presentan una acción sinérgica. Las comparaciones ortogonales dentro del tratamiento "orquidectomía y atrazina + progesterona", a diferencia del tratamiento "orquidectomía y progesterona", no arrojaron diferencias significativas al ser comparado con los demás tratamientos; similar a lo que ocurrió en el tratamiento atrazina. Sin embargo, se pueden encontrar un mayor número oocitos de estadio mayores de oogénesis (III y IV), lo que indica que posiblemente la hormona progesterona es la que indujo el crecimiento en los oocitos mencionados. De igual manera, al comparar el diámetro máximo promedio de los oocitos del tratamiento "orquidectomía y progesterona" $(358 \mu \mathrm{m})$ con los diámetros máximos promedios de los otros tratamientos se puede observar que se produjo un crecimiento en los oocitos del OB de los machos pertenecientes a este tratamiento, pero que dicho crecimiento no se compara con lo obtenido en el tratamiento progesterona. Dichas aseveraciones no se pueden corroborar debido a que estos dos compuestos nunca han sido probados de manera conjunta en estudios anteriores. 


\section{REFERENCIAS BIBLIOGRÁFICAS}

Bill S. 2002. Developmental biology: An arresting activity. Nature, 416: $804-805$.

Blood D, Studdert V y Gay C. 2007. Saunders Comprehensive Veterinary Dictionary. 3th Edition. Saunders Elsevier. Edinburgh-Scotland. 102 pp.

Brown F, del Pino ME y Krohne G. 2002. Bidder's organ in the toad Bufo marinus: Effects of orchidectomy on the morphology and expression of lamina-associated polypeptide 2. Development, Growth and Differentiation, 44: 527-535.

Browne R, Li H, Seratt J y Kouba A. 2006. Progesterone improves the number and quality of hormone induced Fowler toad (Bufo fowleri) oocytes. Reproductive Biology and Endocrinology, 4: 3.

Browne R, Li H, Seratt J, Vance C y Kouba A. 2006. Hormonal priming, induction of ovulation and in-vitro fertilization of the endangered Wyoming toad (Bufo baxteri). Reproductive Biology and Endocrinology, 4: 34.

Cannatella D, Ford L y Bockstanz L. 2001. Neobatrachia: "higher" frogs. University of Arizona. Página de Internet: http:// phylogeny.arizona.edu/tree/eukaryotes/ animals/chordata/salientia/neobatrachia. htl. Consultada: 17-febrero-2010.

Davis D. 1936. The distribution of Bidder's organ in the Bufonidae. Zoological series of Field Museum of Natural HistoryZoology, 20: 115-125.

Diamanti-Kandarakis E, Bourguignon J, Giudice L, Hauser R, Prins G, Soto A, Zoeller T y Gore A. 2009. Endocrine-Disrupting Chemicals: An Endocrine Society Scientific Statement. Endocrine Reviews, 30: 293-342.

Duellman W y Trueb L. 1986. Biology of Amphibians. McGraw-Hill Publishing Company. Baltimore-USA. 472 pp.

Dumont J y Brummett A. 1978. Oogénesis in Xenopus laevis (Daudin). V. Relationships between developing oocytes and their investing follicular tissues. Journal of Morphology, 155: 73-98.
Falconi R, Dalpiaz D y Zaccanti F. 2004. U1trastructural Aspects of Gonadal Morphogenesis in Bufo bufo (Amphibia Anura) 1. Sex Differentiation. Journal of Experimental Zoology, 301: 378-388.

Falconi R, Dalpiaz D y Zaccanti F. 2007. Morphological Aspects of Gonadal Morphogenesis in Bufo bufo (Amphibia Anura): Bidder's Organ Differentiation. The Anatomical Record, 290: 801-813.

Farias C, Carvalho-E-Silva S y de Brito-Gitirana L. 2002. Bidder's organ of Bufo ictericus: a light and electron microscopy analysis. Micron, 33: 673-679.

Frost DR, Grant T, Faivovich J, Bain RH, Haas A, Haddad CFB, de Sá RO, Channing A, Wilkinson $\mathrm{M}$, Donnellan SC, Raxworthy CJ, Campbell JA, Blotto BL, Moler P, Drewes RC, Nussbaum RA, Lynch JD, Green DM y Wheeler WC. 2006. The amphibian tree of life. Bulletin of the American Museum of Natural History, 297: 1-370.

Ghosh A, Ghosh D, Ghosh P y Biswas N. 1990. Influence of pituitary on histology of Bidder's organ in castrated toad Bufo melanostictus. Indian Journal of Experimental Biology, 28: 790-791.

Gilbert S. 2010. Developmental Biology. Ninth Edition. Sinauer Associates, Inc., Publishers. Massachusetts-USA. 603-604 pp.

Hausen P y Riebesell M. 1991. The early development of Xenopus laevis: An Atlas of the Histology. Springer-Verlag. Berlin-Germany.

Hayes T, Anderson L, Beasley V, Solla S, Iguchi $\mathrm{T}$, Ingraham $\mathrm{H}$, Kestemont $\mathrm{P}$, Kniewald J, Kniewald Z, Langlois V, Luque E, Mccoyk K, Muñoz-de-Toro M, Oka T, Oliveira C, Orton F, Ruby S, Suzawa M, Tavera-Mendoza L, Trudeau V, Victor-Costa A y Willingham E. 2011. Demasculinization and feminization of male gonads by atrazine: Consistent effects across vertebrate classes. The Journal of Steroid Biochemistry and Molecular Biology, 127: 64-73. 
Hayes T, Collins A, Lee M, Mendoza M, Noriega N, Stuart A y Vonk A. 2002. Hermaphroditic, demasculinized frogs after exposure to the herbicide atrazine at low ecologically relevant doses. Proceedings of the National Academy of Sciences, 99: $5476-5480$.

Hayes T, Haston K, Tsui M, Hoang A, Haeffele C y Vonk A. 2003. Atrazine-Induced Hermaphroditism at $0.1 \mathrm{ppb}$ in American Leopard Frogs (Rana pipiens): Laboratory and Field Evidence. Environmental Health Perspectives, 111: 568-575.

Hayes T, Khoury V, Narayan A, Nazir M, Park A, Brown T, Adame L, Chan E, Buchholz D, Stueve T, Gallipeau S. 2010. Atrazine induces complete feminization and chemical castration in male African clawed frogs (Xenopus laevis). Proceedings of the National Academy of Sciences, 107: 4612-4617.

Hayes T. 1998. Sex Determination and Primary Sex Differentiation in Amphibians: Genetic and Developmental Mechanisms. The Journal of Experimental Zoology, 281: 373-399.

Lee L. 1960. Manual of Histologic Staining Methods of the Armed Forces Institute of Pathology. 3th Edition. McGraw Hill Publishers. New York-USA. 56 pp.

Liu J. 2006. Xenopus protocols: cell biology and signal transduction. Human Press. New Jersey-USA. 84 pp.

McCoy K, Bortnick J, Campbell C, Hamlin H, Guillette L y Colette M. 2008. Agriculture Alters Gonadal Form and Function in the Toad Bufo marinus. Environmental Health Perspectives 116: 1526-1532.

Norris D y Lopez K. 2011. Hormones and Reproduction of Vertebrates. Volume 2: Amphibians. Elsevier Inc. San DiegoUSA. 6-10, 55-59, 206 pp.

Pancak-Roessler M y Norris D. 1991. The Effects of Orchidectomy and Gonadotropins on Steroidogenesis and Oogenesis in Bidder's Organs of the Toad Bufo woodhousii. The Journal of Experimental Zoology, 260: 323-336.
Pramuk J. 2006. Phylogeny of South American Bufo (Anura: Bufonidae) inferred from combined evidence. Zoological Journal of the Linnean Society, 146: 407-452.

Pramuk JB, Robertson JB, Sites JW y Noonan BP. 2008. Around the world in 10 million years: biogeography of the nearly cosmopolitan true toads (Anura: Bufonidae). Global Ecology and Biogeography, 17: 72-83.

Schuetz A. 1974. Role of Hormones in Oocyte Maturation. Biology of Reproduction, 10: 150-178.

Takahashi H. 1956. The Effects of Some Sex Hormones on the Development of Bidder's Organ in Bufo vulgaris formosus Boulenger. Journal of the faculty of science Hokkaido University, 12: 297-308.

Wallace H, Badawy G y Wallace B. 1999. Amphibian sex determination and sex reversal. Cellular and Molecular Life Sciences, 55: 901-909.

Wallace R y Bergink E. 1974. Amphibian Vitellogenin: Properties, Hormonal Regulation of Hepatic Synthesis and Ovarian Uptake, and Conversion to Yolk Proteins. American Zoologist, 14: 1159-1175.

Wallace R y Selman K. 1990. Ultrastructural aspects of oogenesis and oocyte growth in fish and amphibians. Journal of Electron Microscopy Technique, 16:175-201.

Wolpert L, Jessell T, Lawrence P, Meyerowitz E, Robertson E y Smith J. 2007. Principles of Development. Third Edition. Oxford University Press. New YorkUSA, pp 96-99.

Zaccanti F y Tognato G. 1976. Effects of different doses of diethylstilbestrol dipropionate on the Bidder's organ of intact or orchidectomized adult males of Bufo bufo. Monitore Zoology, 10:105-117.

Zug G y Zug P. 1979. The Marine Toad, Bufo marinus. A Natural History Resume of Native Populations. Smithsonian Institution Press. Washington-USA. 8 pp. 